\title{
New spectro-photometric characterization of the substellar object HR 2562 B using SPHERE`
}

\author{
D. Mesa ${ }^{1,2}$, J.-L. Baudino ${ }^{3}$, B. Charnay ${ }^{4}$, V. D’Orazi ${ }^{1}$, S. Desidera $^{1}$, A. Boccaletti ${ }^{4}$, R. Gratton ${ }^{1}$, \\ M. Bonnefoy ${ }^{6}$, P. Delorme ${ }^{6}$, M. Langlois ${ }^{7,8}$, A. Vigan ${ }^{8}$, A. Zurlo ${ }^{9,10,8}$, A.-L. Maire ${ }^{11}$, M. Janson ${ }^{11,12}$, \\ J. Antichi ${ }^{1,13}$, A. Baruffolo ${ }^{1}$, P. Bruno ${ }^{14}$, E. Cascone ${ }^{15}$, G. Chauvin ${ }^{6,16}$, R.U. Claudi ${ }^{1}$, V. De Caprio ${ }^{15}$, \\ D. Fantinel ${ }^{1}$, G. Farisato ${ }^{1}$, M. Feldt ${ }^{11}$, E. Giro ${ }^{1,17}$, J. Hagelberg ${ }^{6}$, S. Incorvaia ${ }^{18}$, E. Lagadec ${ }^{19}$, \\ A.-M. Lagrange ${ }^{6}$, C. Lazzoni ${ }^{1}$, L. Lessio ${ }^{1}$, B. Salasnich ${ }^{1}$, S. Scuderi ${ }^{14}$, E. Sissa ${ }^{1}$, and M. Turatto ${ }^{1}$ \\ ${ }^{1}$ INAF-Osservatorio Astronomico di Padova, Vicolo dell'Osservatorio 5, Padova 35122, Italy \\ e-mail: dino.mesa@oapd.inaf.it \\ 2 INCT, Universidad De Atacama, calle Copayapu 485, Copiapó, Atacama, Chile \\ ${ }^{3}$ Department of Physics, University of Oxford, Oxford, UK \\ ${ }^{4}$ LESIA, Observatoire de Paris, PSL Research University, CNRS, Sorbonne Universités, UPMC Univ. Paris 06, Univ. Paris Diderot, \\ Sorbonne Paris Cité, 5 Place Jules Janssen, 92195 Meudon, France \\ 5 Virtual Planetary Laboratory, University of Washington, Seattle, WA 98125, USA \\ ${ }^{6}$ Univ. Grenoble Alpes, IPAG; CNRS, IPAG, 38000 Grenoble, France \\ 7 Univ. Lyon, Univ. Lyon 1, ENS de Lyon, CNRS, CRAL UMR 5574, 69230 Saint-Genis-Laval, France \\ 8 Aix-Marseille Université, CNRS, LAM “Laboratoire d'Astrophysique de Marseille”, UMR 7326, 13388 Marseille, France \\ ${ }^{9}$ Nucleo de Astronomía, Facultad de Ingeniería, Universidad Diego Portales, Av. Ejercito 441, Santiago, Chile \\ ${ }^{10}$ Universidad de Chile, Camino el Observatorio, 1515 Santiago, Chile \\ ${ }_{11}^{11}$ Max-Planck-Institut für Astronomie, Känigstuhl 17, 69117 Heidelberg, Germany \\ ${ }^{12}$ Department of Astronomy, Stockholm University, 10691 Stockholm, Sweden \\ ${ }^{13}$ INAF Osservatorio Astrofisico di Arcetri, Largo E. Fermi 5, 50125 Firenze, Italy \\ ${ }^{14}$ INAF Osservatorio Astrofisico di Catania, Via S. Sofia 78, 95123 Catania, Italy \\ 15 INAF Osservatorio Astronomico di Capodimonte, Via Moiariello 16, 80131 Napoli, Italy \\ ${ }^{16}$ Unidad Mixta Internacional Franco-Chilena de Astronomía, CNRS/INSU UMI 3386 and Departamento de Astronomía, Universidad \\ de Chile, Casilla 36-D, Santiago, Chile \\ ${ }^{17}$ INAF Osservatorio Astronomico di Brera, via Emilio Bianchi 46, 23807 Merate (LC), Italy \\ ${ }^{18}$ INAF Istituto di Astrofisica Spaziale e Fisica Cosmica di Milano, Via E. Bassini 15, 20133 Milano, Italy \\ ${ }^{19}$ Université Côte d'Azur, OCA, CNRS, Lagrange, France
}

Received 26 July 2017 / Accepted 14 December 2017

\begin{abstract}
Aims. HR 2562 is an F5V star located at $\sim 33$ pc from the Sun hosting a substellar companion that was discovered using the Gemini planet imager (GPI) instrument. The main objective of the present paper is to provide an extensive characterization of the substellar companion, by deriving its fundamental properties.

Methods. We observed HR 2562 with the near-infrared branch composed by the integral field spectrograph (IFS) and the infrared dual band spectrograph (IRDIS) of the spectro-polarimetric high-contrast exoplanet research (SPHERE) instrument at the very large telescope (VLT). During our observations IFS was operating in the $Y J$ band, while IRDIS was observing with the $H$ broadband filter. The data were reduced with the dedicated SPHERE GTO pipeline, which is custom designed for this instrument. On the reduced images, we then applied the post-processing procedures that are specifically prepared to subtract the speckle noise.

Results. The companion is clearly detected in both IRDIS and IFS datasets. We obtained photometry in three different spectral bands. The comparison with template spectra allowed us to derive a spectral type of T2-T3 for the companion. Using both evolutionary and atmospheric models we inferred the main physical parameters of the companion obtaining a mass of $32 \pm 14 M_{\mathrm{Jup}}, T_{\text {eff }}=1100 \pm 200 \mathrm{~K}$, and $\log g=4.75 \pm 0.41$.
\end{abstract}

Key words. instrumentation: spectrographs - methods: data analysis - techniques: imaging spectroscopy - planetary systems stars: late-type

\section{Introduction}

In the last decade, the research field focussed on the atmospheric characterization of bound substellar objects (i.e., brown dwarfs

^ Based on observations made with European Southern Observatory (ESO) telescopes at Paranal Observatory in Chile, under program ID 198.C-0209(D). and giant planets) has experienced an outstanding boost. In fact, high-contrast imaging observations have permitted the discovery of an increasing number of substellar companions (Chauvin et al. 2004, 2005; Marois et al. 2008; Lagrange et al. 2010; Biller et al. 2010; Rameau et al. 2013; Bailey et al. 2014; Macintosh et al. 2015; Gauza et al. 2015; Milli et al. 2017; Bowler et al. 2017). In particular, the new generation of extreme adaptive optics (XAO) 
high-contrast imaging facilities such as the spectro-polarimetric high-contrast exoplanet research (SPHERE) instrument at the very large telescope (VLT; Beuzit et al. 2008) and the Gemini planet imager (GPI) at Gemini (Macintosh et al. 2014) have proven to be remarkably efficient for this purpose. Several recent examples of spectroscopic characterization of substellar companions with these instruments include HD 95086b (De Rosa et al. 2016), 51 Eri b (Macintosh et al. 2015; Samland et al. 2017), $\beta$ Pic b (Chilcote et al. 2017), HD 1160 B (Maire et al. 2016; Garcia et al. 2017), HD 984 B (Johnson-Groh et al. 2017), GJ 504 b (Bonnefoy 2015), GJ 758 B (Vigan et al. 2016), the four planets of the HR 8799 system (Bonnefoy et al. 2016; Zurlo et al. 2016), and HR 3549 B (Mesa et al. 2016).

HR 2562 (HIP 32775; HD 50571) is an F5V star with an estimated mass of $M \sim 1.3 M_{\odot}$ and a distance of $d=33.63 \pm 0.48$ pc (see Sect. 2). Moór et al. (2006) identified a debris disk around it by exploiting IRAS and Spitzer data. Chen et al. (2014) modeled the stellar spectral energy distribution (SED) with a two-component disk placed at 1.1 and $341.6 \mathrm{au}$, respectively. More recently, Moór et al. (2015), using Herschel data, derived a dust radius of $112.1 \pm 8.4$ au with evidence of an inner hole with a radius between 18 and $70 \mathrm{au}$, and a high inclination of $78.0^{\circ} \pm 6.3^{\circ}$. Konopacky et al. (2016) found a substellar companion with mass in the brown dwarf regime of $30 \pm 15 M_{\text {Jup }}$ and effective temperature $T_{\text {eff }}=1200 \pm 100 \mathrm{~K}$. Its separation of $\sim 0.6^{\prime \prime}$ corresponding to $\sim 20$ au from the star put it into the hole in the disk and its separation and position angle are compatible with its orbit being coplanar with the disk itself.

HR 2562 was observed with SPHERE in order to perform a thorough investigation of the spectral properties of its substellar companion, to constrain the physical parameters and fundamental properties, and to obtain new highprecision astrometric data to better define its orbit and the relation with the disk. In this paper we discuss the spectrophotometric data obtained for this target, whereas the astrometric data will be discussed in a second paper (Maire et al., in prep.).

The paper is organized as follows: in Sect. 2 we discuss the main physical characteristics of the host star, in Sect. 3 we describe the observations and data reduction, in Sect. 4 we report the results that are then discussed in Sect. 5, and in Sect. 6 we provide our conclusions.

\section{Host star properties}

A careful determination of the fundamental parameters of the host star is crucial in order to obtain reliable and robust estimates of the substellar companion properties. In particular, the most crucial issue is the stellar age that is poorly defined for HR 2562, as it is for mid F-type stars in general. Asiain et al. (1999) defined an age of $300 \pm 120$ Myr classifying HR 2562 as a member of the B3 group. A similar age of $\sim 300 \mathrm{Myr}$ was subsequently determined by Rhee et al. (2007) using space motions, lithium nondetection, and X-ray luminosity. Exploiting the metallicity and the temperature from the Geneva-Copenaghen survey, Casagrande et al. (2011) defined a much older age of 0.9-1.6 Gyr and a similar result of $\sim 0.9$ Gyr based on chromospheric activity was found by Pace (2013). Finally, Moór et al. (2011) derived an age range of $300_{-180}^{+420} \mathrm{Myr}$ using evolutionary models. We tried to determine the main stellar parameters following the methods described in Desidera et al. (2015), but more specifically tuned for a mid-F star as previously done for HD 206893 B in Delorme et al. (2017).

\subsection{Spectroscopic parameters}

In order to carry out a spectroscopic analysis of HR 2562 (aiming at deriving radial and rotational velocities, along with atmospheric parameters, $T_{\text {eff }}, \log g$, and metallicity $\left.[\mathrm{Fe} / \mathrm{H}]\right)$, we retrieved from the ESO archive two UVES spectra ${ }^{1}$ acquired on the same night, and two FEROS spectra ${ }^{2}$ separated by a few months. In all cases, data reduction was performed using the online pipeline tools provided by ESO.

From these spectra we measured the radial velocity (RV) and the projected rotation velocities $(v \sin i)$ (Table 1$)$ using a custom cross-correlation function (CCF) procedure. While our radial and projected rotational velocity estimates agree fairly well with each other, there is a significant discrepancy (about $6 \mathrm{~km} \mathrm{~s}^{-1}$ in RV and about $25 \mathrm{~km} \mathrm{~s}^{-1}$ in $v \sin$ ) with the results obtained with CORAVEL (Andersen et al. 1985; de Medeiros \& Mayor 1999; Nordström et al. 2004). These differences can be explained if the star is an SB2 system with the components seen at similar velocities at the epoch of FEROS and UVES observations. However, this hypothesis has several difficulties. There are no significant RV differences between the FEROS and UVES epochs and the two CORAVEL observations, suggesting a moderately long period, but the period should be short enough to ensure a RV amplitude large enough to explain the $v \sin i$ variations. Also, the acquisition images obtained with SPHERE do not show the presence of equal luminosity companions down to separation of about 40 mas. Therefore, the projected separation at the epoch of the SPHERE observations would have been smaller than about $1.35 \mathrm{au}$. All these constraints can only be satisfied by a very narrow range of binary parameters. Furthermore, for an SB2 system with similar components (required to explain a variable FWHM of the CCF) an unphysical position on the color-magnitude diagram below the main sequence is obtained. We then conclude that additional observations are needed to settle the issue of the binarity of the central star.

In agreement with Asiain et al. (1999), we confirm that the kinematic parameters of the star are similar to those of the B3 group when adopting the Andersen et al. (1985) RV; instead, they deviate significantly when adopting our own RV determination. Concerning the younger groups, no significant membership probability is returned using the online BANYAN II tool (Gagné et al. 2014) for both values of system RV. Taking the uncertainties in system RV into account, we do not consider the kinematic as constraining the stellar age in the following.

The star was also detected by ROSAT, yielding $\log L_{\mathrm{X}} / L_{\mathrm{bol}}=-5.21 \pm 0.06$. This value is within the locus of Hyades stars (Fig. 1, panel $c$ ). The chromospheric emission parameter obtained by Gray et al. (2006) $\log R_{\mathrm{HK}}=-4.55$ (Fig. 1, panel $b$ ) is likely overestimated (see Desidera et al. 2015) and cannot give any constraints on the stellar parameters.

The highest quality FEROS spectrum (median signal-tonoise ratio $S / N=305$ per pixel) was also used to derive the stellar parameters. The moderately fast rotation makes it challenging to perform a standard abundance analysis. Selecting 14 FeI and 2 Fe II isolated lines, we derived $T_{\text {eff }}=6650 \pm 100 \mathrm{~K}$, $\log g=4.3 \pm 0.2$, and $[\mathrm{Fe} / \mathrm{H}]=+0.13 \pm 0.02$ (internal error due to line-by-line scatter) and microturbulence $\xi=1.8 \mathrm{~km} \mathrm{~s}^{-1}$. The same temperature value is obtained by fitting the wings of the $H_{\alpha}$ line, employing the SME code (Valenti \& Piskunov 1996).

\footnotetext{
1 Prog. ID 096.C-0238(A)

2 Prog. ID 094.A-9012(A)
} 
Table 1. Radial velocity and projected rotational velocity

\begin{tabular}{lccll}
\hline \hline Date & $\mathrm{RV}\left(\mathrm{km} \mathrm{s}^{-1}\right)$ & $v \sin i\left(\mathrm{~km} \mathrm{~s}^{-1}\right)$ & Instrument & Reference \\
\hline $2008-06-14$ & $22.90 \pm 0.81$ & 60.0 & CORAVEL & Andersen et al. (1985); Nordström et al. (2004) \\
$2009-05-29$ & $21.58 \pm 0.80$ & 60.0 & CORAVEL & Andersen et al. (1985); Nordström et al. (2004) \\
$2016-02-23$ & $29.28 \pm 0.40$ & 31.3 & UVES & This paper \\
$2016-03-26$ & $27.72 \pm 0.43$ & 35.0 & FEROS & This paper \\
\hline
\end{tabular}
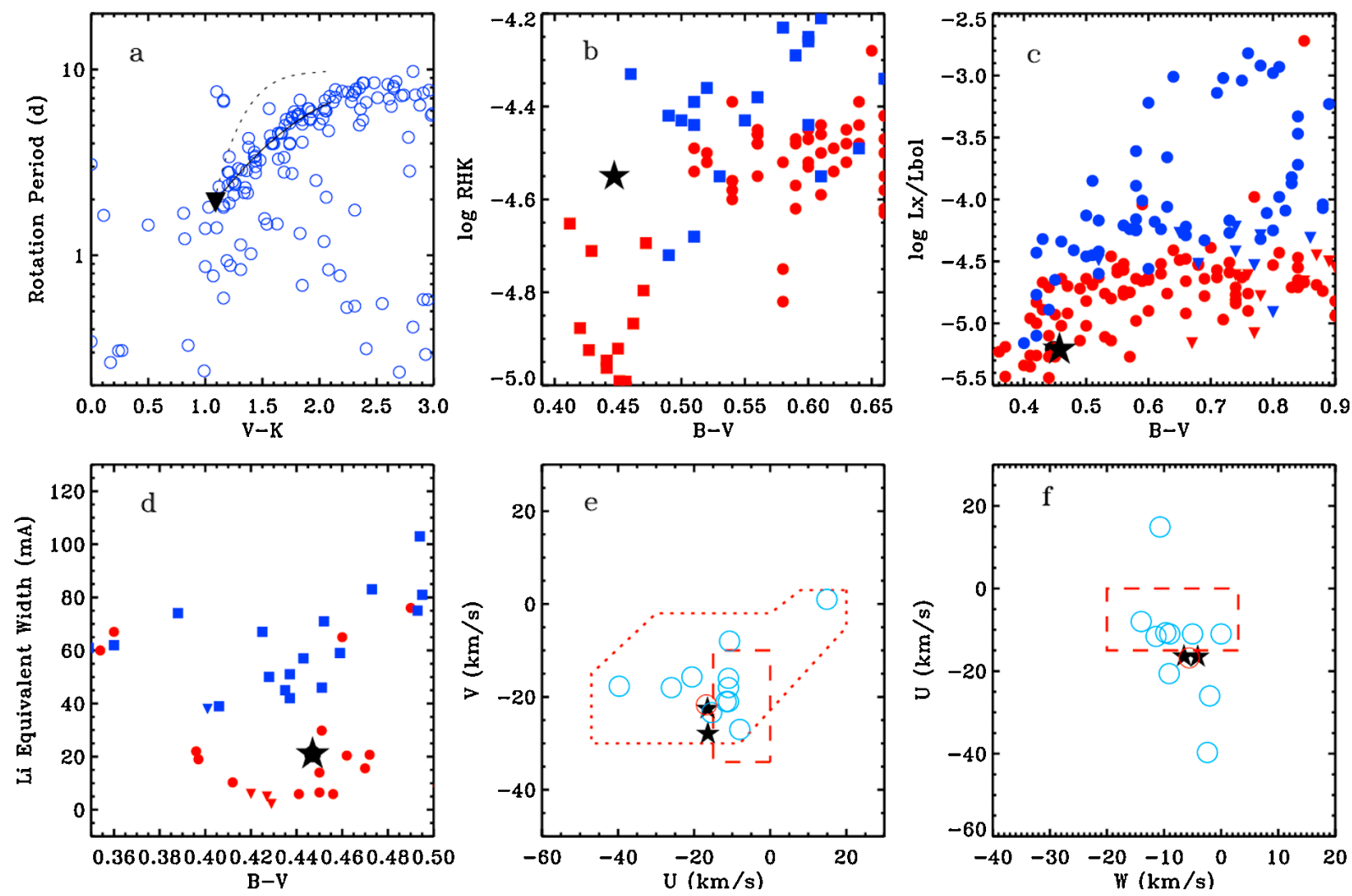

Fig. 1. Comparison of rotation period, chromospheric emission $\log R_{\mathrm{HK}}$, coronal emission $\log L_{\mathrm{X}} / L_{\mathrm{bol}}$, and Li EW of HR 2562 to those of Hyades (red symbols) and Pleiades (blue symbols) open clusters. In panel a (rotation period), the triangle represents the upper limits derived from the projected rotational velocity. In the same panel the continuous and dashed lines are the sequences of slow rotators for Pleiades and Praesepe, respectively (Stauffer et al. 2016). In panels $b, c$, and $d$, showing respectively the chromospheric emission, $\log L_{\mathrm{X}} / L_{\mathrm{bol}}$, and lithium equivalent width, circles and squares represent measurements, while upsidedown triangles represent upper limits. Panels $e$ and $f$ show the kinematic parameters of HR 2562 for the two values of RV derived in this paper and from CORAVEL data, compared to those of several young moving groups. In these panels, the red open circle represents the B3 MG, while the dotted and dashed lines show the limits of the young disk as defined by Montes et al. (2001) and the young star box as defined by Zuckerman \& Song (2004), respectively.

The mild metal overabundance is also supported by Strömgren photometry (Casagrande et al. 2011) with $[\mathrm{Fe} / \mathrm{H}]=+0.07$. The effective temperature derived in that study is $6597 \pm 81 \mathrm{~K}$. Considering the uncertainties in the spectral analysis for such a moderately fast-rotator mid-F star, we adopt this latter value. Repeating the abundance analysis for the adopted $T_{\text {eff }}$ yields $[\mathrm{Fe} / \mathrm{H}]=+0.10 \pm 0.02$.

Isochrone stellar age and stellar mass were derived exploiting the Bressan et al. (2012) stellar models and the PARAM interface (da Silva et al. 2006) with the input parameters above. The stellar age is not well constrained $(630 \pm 540 \mathrm{Myr})$, as expected for a star that lies close to the zero age main sequence (ZAMS). HR 2562 is not known as a variable star and HIPPARCOS photometry shows a scatter of only 0.007 mag from 118 photometric measurements along the mission lifetime. Therefore, it is unlikely that variability affects the isochrone ages significantly.

\subsection{Lithium abundance}

The FEROS spectrum was also exploited to measure the lithium content, a key age indicator for mid-F stars thanks to the presence of the Li dip feature (Boesgaard \& Tripicco 1986). Indeed, a marked drop in lithium abundance is seen for stars in a narrow range around $T_{\text {eff }} 6660 \mathrm{~K}$. The lithium dip is clearly observed in the Hyades and other older open clusters (Balachandran 1995); it starts to be present at the age of the open cluster M35 (Steinhauer \& Deliyannis 2004), but it is not seen in the Pleiades and for younger clusters (Boesgaard et al. 1988). We performed the first measurement of lithium content in HR 2562, obtaining EW Li $=21 \pm 5 \mathrm{~m} \AA$ and, through spectral synthesis as described in Desidera et al. (2011) and D'Orazi et al. (2017), $\mathrm{A}(\mathrm{Li})=2.55 \pm 0.2$. As for HIP 107412, which has similar $T_{\text {eff }}$ (Delorme et al. 2017), lithium provides the tightest constraints on the stellar age. Comparison with 
Table 2. Stellar parameters of HR 2562.

\begin{tabular}{lcl}
\hline \hline Parameter & Value & Ref \\
\hline$V$ (mag) & 6.11 & HIPPARCOS \\
$B-V(\mathrm{mag})$ & 0.457 & HIPPARCOS \\
$V-I(\mathrm{mag})$ & 0.53 & HIPPARCOS \\
$J(\mathrm{mag})$ & $5.305 \pm 0.020$ & 2MASS \\
$H(\mathrm{mag})$ & $5.128 \pm 0.029$ & 2MASS \\
$K(\mathrm{mag})$ & $5.020 \pm 0.016$ & 2MASS \\
Parallax $(\mathrm{mas})$ & $29.73 \pm 0.40$ & GaiaDR1 \\
$\mu_{\alpha}\left(\mathrm{mas} \mathrm{yr}^{-1}\right)$ & $4.872 \pm 0.040$ & GaiaDR1 \\
$\mu_{\delta}\left(\mathrm{mas} \mathrm{yr} \mathrm{y}^{-1}\right)$ & $108.568 \pm 0.040$ & GaiaDR1 \\
$\left.\mathrm{RV}(\mathrm{km} \mathrm{s})^{-1}\right)$ & $27.72 \pm 0.43$ & This paper \\
$T_{\text {eff }}(\mathrm{K})$ & $6597 \pm 81$ & Casagrande et al. $(2011)$ \\
$\log g$ & $4.3 \pm 0.2$ & This paper \\
{$[\mathrm{Fe} / \mathrm{H}]$} & $0.10 \pm 0.06$ & This paper \\
EW Li $\left(\mathrm{m} \AA^{\circ}\right)$ & $21 \pm 5$ & This paper \\
$\mathrm{A}(\mathrm{Li})$ & $2.55 \pm 0.20$ & This paper \\
$v \sin i\left(\mathrm{~km} \mathrm{~s}^{-1}\right)$ & $33.2 \pm 2.6$ & This paper \\
$\log L_{\mathrm{X}} / L_{\mathrm{bol}}$ & $-5.21 \pm 0.06$ & This paper \\
Age $(\mathrm{Myr})$ & $200-750$ & This paper \\
$M_{\text {star }}\left(M_{\odot}\right)$ & $1.368 \pm 0.018$ & This paper \\
$R_{\text {star }}\left(R_{\odot}\right)$ & $1.334 \pm 0.027$ & This paper \\
\hline & &
\end{tabular}

lithium observed in other intermediate age clusters and associations indicates that the lithium content of HR 2562 is close to the upper boundaries of the locus of the Hyades, close to the lower boundaries of that of M35 (Steinhauer \& Deliyannis 2004) and well below the locus of the Pleiades (see Fig. 1, panel $d$ ). While the scatter in the $\mathrm{Li}-T_{e} f f$ relationship is significant for all the clusters, preventing a well-defined age calibration, this result confines the age of the star between 200 and 750 Myr. In the following we will consider these age limits in the derivation of the companion properties, and $450 \mathrm{Myr}$ as the representative intermediate value. This age estimate is consistent with (but more constraining than) the other dating techniques presented above and is slightly narrower than that adopted by Konopacky et al. (2016). The stellar mass and radius derived as above, although allowing only this age range (see Desidera et al. 2015), are $1.368 \pm 0.018 M_{\odot}$ and $1.334 \pm 0.027 R_{\odot}$, respectively.

\section{Observations and data reduction}

HR 2562 was observed with SPHERE on February 6, 2017, using a nonstandard configuration for the IRDIFS mode. In this case, the integral field spectrograph (IFS; Claudi et al. 2008) operated in the $Y$ and $J$ bands between 0.95 and $1.35 \mu \mathrm{m}$ while the infrared dual band spectrograph (IRDIS; Dohlen et al. 2008) used the $\mathrm{H}$ broadband filter configuration instead of the standard dual-band configuration in H2-H3 filters (Vigan et al. 2010). This choice was done in order to image the disk. The IFS data were composed of 16 datacubes of 5 frames with an exposure time of $64 \mathrm{~s}$; those of IRDIS were composed of 16 datacubes of 20 frames with an exposure time of 16 s. The IRDIS observations were performed using a $4 \times 4$ dithering pattern, while no dithering was used for IFS. To be able to use the angular differential imaging (ADI; Marois et al. 2006a) technique, the field of view (FOV) was allowed to rotate during the observations, with the pupil fixed with respect to the detector. To maximize the total rotation of the FOV, we observed the star during its passage to the meridian with a total rotation of $\sim 34.9^{\circ}$. For both IFS and IRDIS we acquired observing frames with the star image off-centered with respect to the coronagraph to be able to calibrate the companion flux. In order to avoid saturation, a neutral density filter was employed. Moreover, we took frames with four satellite spots that are symmetrically located with respect to the central star by a suitable modification of the wavefront provided by the AO. This is done to properly determine the center of each frame: the use of satellite spots was first proposed by Sivaramakrishnan \& Oppenheimer (2006) and Marois et al. (2006b). We refer to Langlois et al. 2013 and Mesa et al. 2015 for an extended discussion of their use within the SPHERE framework.

Both IFS and IRDIS data were reduced using the SPHERE data center ${ }^{3}$ applying the appropriate calibrations following the SPHERE data reduction and handling (DRH; Pavlov et al. 2008) pipeline. More specifically, the IRDIS dataset requires the application of dark and flat-field frames and the definition of the star center. On the other hand, for IFS observations the reduction steps include dark and flat-filed correction, definition of the spectral positions, wavelength calibration, and the application of the instrumental flat. For IFS, on the wavelength-calibrated datacubes (each composed of 39 monochromatic frames), we then applied the speckle subtraction exploiting the principal components analysis (PCA; Soummer et al. 2012) as described in Mesa et al. (2015) and Zurlo et al. (2014). For IRDIS we performed the speckle subtraction with both PCA and TLOCI (Marois et al. 2014) using the consortium pipeline (Galicher et al., in prep.).

\section{Results}

The data reduction procedure described in Sect. 3 provided the IFS and IRDIS final images displayed in Fig. 2. The companion is clearly detected with both instruments. However, it was not possible to image the debris disk even though we decided to employ the IRDIS $\mathrm{H}$ broadband filter for this specific purpose. The companion was detected with a $\mathrm{S} / \mathrm{N}$ on the order of 30 with IFS, and with a S/N of 20 with IRDIS. In both cases, we exploited the PCA algorithm that allowed us to obtain the best results with respect to other algorithms.

Moreover, we were able to define from the final images the contrast limit for IFS and IRDIS following the procedure devised in Mesa et al. (2015). The contrast values obtained were corrected to take into account the self-subtraction of the highcontrast imaging method. This was evaluated by injecting in the original datacube simulated planets of known flux. The resulting limiting contrast curves are displayed in Fig. 3 where we show that we could obtain a contrast better than $10^{-6}$ at a separation of $\sim 0.3^{\prime \prime}$ or larger with IFS. As for IRDIS, we reach a contrast better than $10^{-7}$ at a separation larger than $1.5^{\prime \prime}$

For the companion, we obtained photometric measurements for each spectral channel introducing a negative simulated planet in the original dataset at the companion position and running our PCA procedure. As a cross-check, we performed the same procedure using a classical ADI procedure and calculated the aperture photometry of the companion on the final images after properly taking into account the self-subtraction. The three methods provide similar results and we list the final photometry obtained using the first method described above for the $Y, J$, and $H$ bands (here and in all the following analysis we use the

\footnotetext{
http://sphere.osug.fr/spip.php?rubrique16\&lang=en
} 

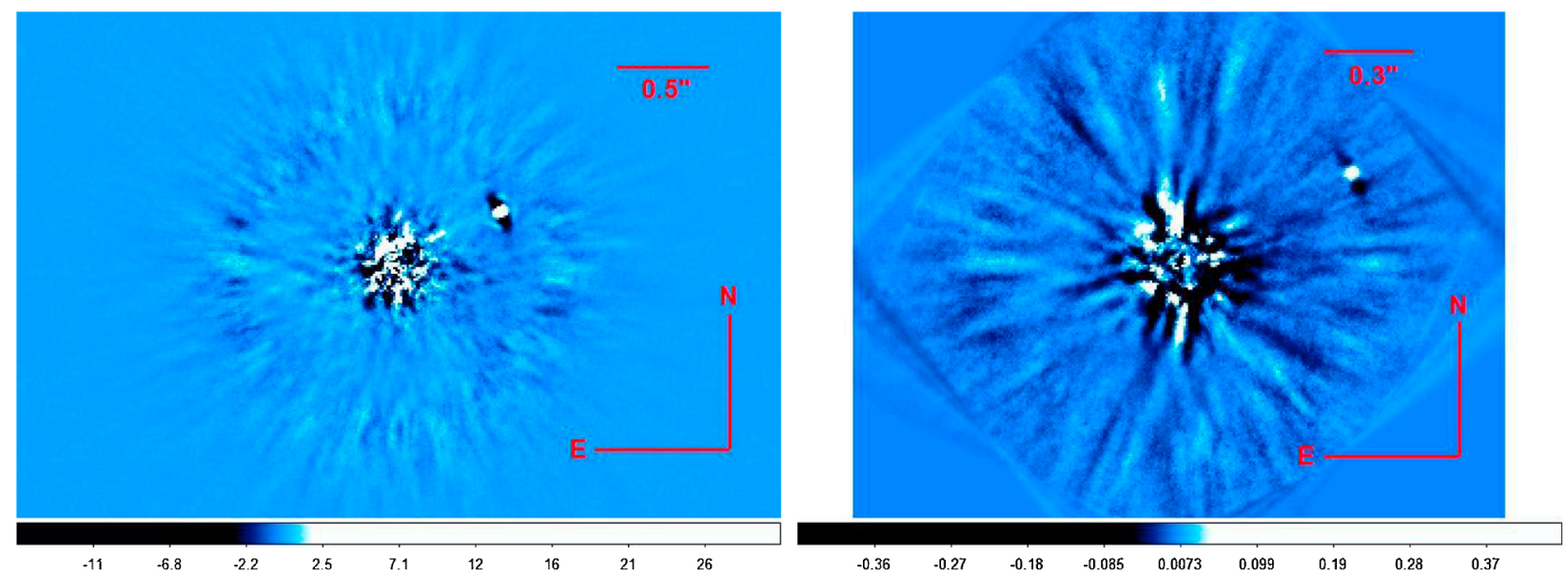

Fig. 2. Left: Final image obtained with IRDIS. Right: Final image obtained with IFS. Both images were obtained using the PCA algorithm.

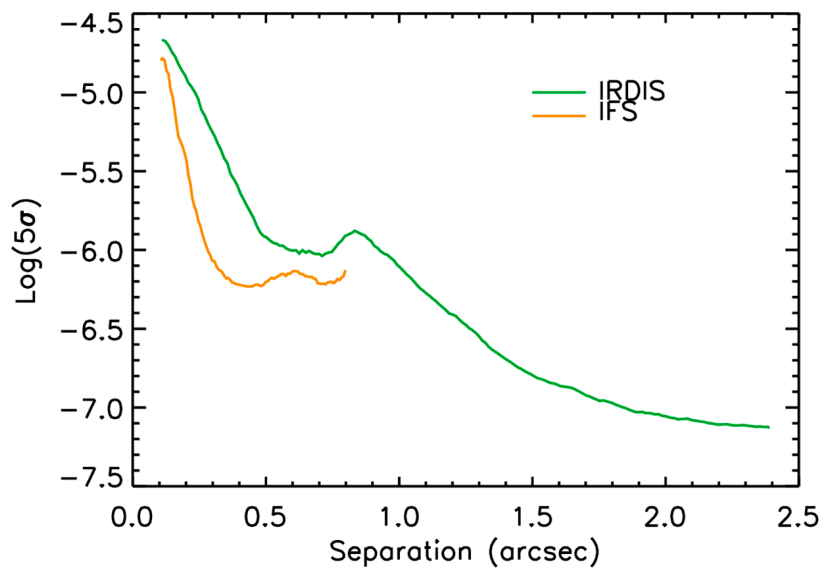

Fig. 3. Contrast plot for IRDIS (green line) and IFS (orange line).

Table 3. Absolute magnitude for HR $2562 \mathrm{~B}$ in $Y, J$, and $H$ band.

\begin{tabular}{ccc}
\hline \hline$M_{Y}$ & $M_{J}$ & $M_{H}$ \\
\hline $15.97 \pm 0.33$ & $15.01 \pm 0.11$ & $13.98 \pm 0.14$ \\
\hline
\end{tabular}

2MASS photometric system) in Table 3. The photometric errors on these values are mainly due to uncertainties on the attenuation factor of the method, to variations on the stellar PSF and to variations in the stellar speckle noise during the observing sequence.

From the photometric data, we obtained a low-resolution spectrum for the companion that was then converted from contrast to flux by multiplying it by a flux-calibrated BT-NEXTGEN (Allard et al. 2012) synthetic spectrum for the host star, adopting $T_{\text {eff }}=6400 \mathrm{~K}, \log g=4.0$, and $[\mathrm{M} / \mathrm{H}]=0.0$, which gives the best fit with the SED of the star. The final result of this procedure is displayed in Fig. 4 and compared with the GPI spectrum from Konopacky et al. (2016). In the $J$ band, the SPHERE IFS and GPI spectra are very similar even if the peak at $\sim 1.27 \mu \mathrm{m}$ is slightly higher ( $\sim 15 \%$, however within the error bars; see Fig. 4) in the SPHERE IFS data. In the $H$ band, in order to compare the IRDIS broadband result with GPI data, we integrated the GPI spectrum over the wavelength range of the IRDIS $\mathrm{H}$ broadband filter. In this case the IRDIS value is about $35 \%$ higher than that from GPI.

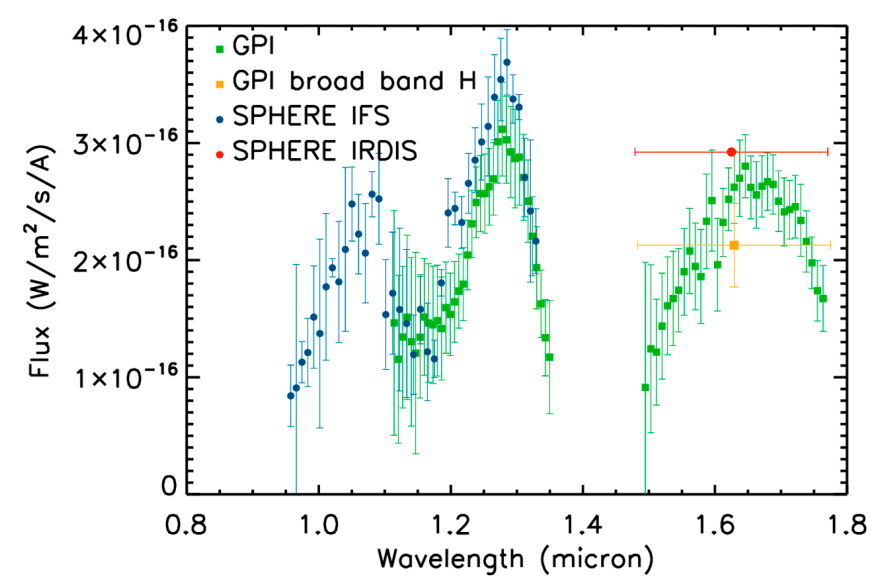

Fig. 4. Low-resolution spectrum for HR 2562 B (blue circles) compared to the GPI spectrum for the same object (green squares). The red circle is the spectral point derived from IRDIS, where the large error band on the wavelength scale denotes that it is derived with a broadband filter. The orange square is derived from the GPI data considering the same wavelength range of the IRDIS broadband filter to be able to make a comparison between the two instruments.

It is noteworthy, however, that systematic errors could be present between GPI and SPHERE probably due to differences in the algorithms for the spectral extraction and/or in the flux normalization procedure, as highlighted by Rajan et al. (2017) who compared the GPI (Macintosh et al. 2015) and SPHERE (Samland et al. 2017) results for 51 Erib. For this reason, in the following analysis we use only the SPHERE data.

\section{Discussion}

\subsection{Characterization of $H R 2562 B$}

\subsubsection{Color-magnitude diagram}

Starting from the photometric values listed in Table 3, we produced the color-magnitude diagram $\left(J_{s}\right.$ vs. $\left.J_{s}-\mathrm{H}\right)$. The use of the $J_{s}$ magnitude is justified in this case because it allows objects near the $\mathrm{L} / \mathrm{T}$ transition to be more easily discriminated from background contaminants. In this diagram, the position of HR $2562 \mathrm{~B}$ is compared to those of M, L, and T field dwarfs, whose photometry has been gathered from the flux-calibrated near-infrared spectra from the SpeXPrism library (Burgasser 2014). As was done in Zurlo et al. (2016), we smoothed these 


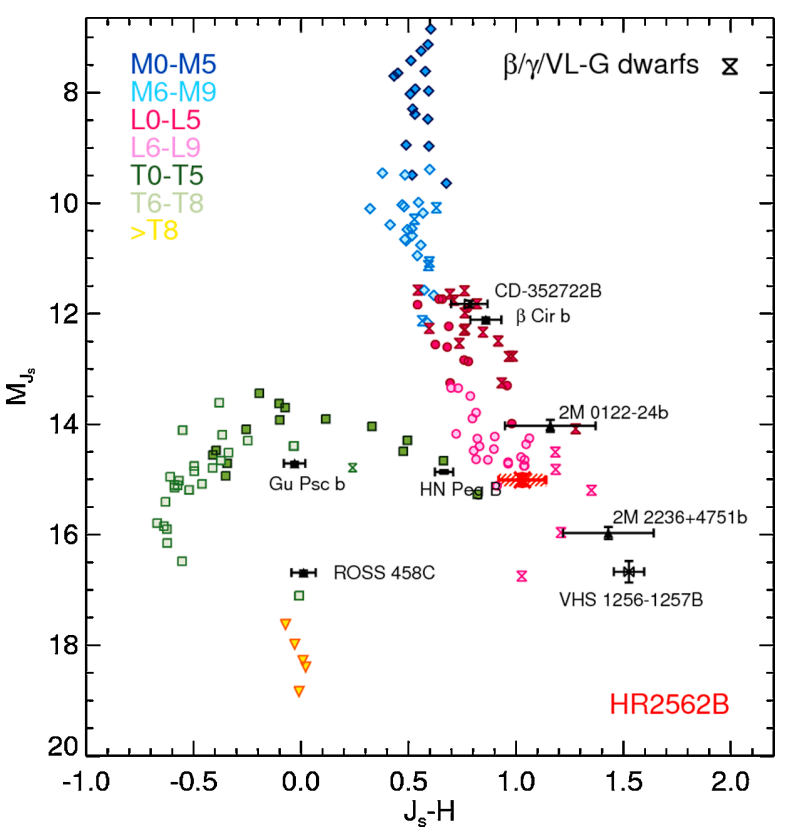

Fig. 5. $J_{s}$ vs. $J_{s}-\mathrm{H}$ diagram showing the position of HR $2562 \mathrm{~B}$, represented by a red star, with respect to that of $\mathrm{M}, \mathrm{L}$, and T field dwarfs and of young known companions. The black asterisks show the positions of substellar companions with an age comparable to that of HR $2562 \mathrm{~B}$, which is indicated by the red point.

spectra to the IRDIS resolution using the IRDIS passbands, a model of the Paranal atmospheric transmission using the ESO Skycalc web application ${ }^{4}$ (Noll et al. 2012; Jones et al. 2013) and a model spectrum of Vega (Bohlin 2007). We also show the positions of substellar companions with an age comparable to that of HR 2562 B (Luhman et al. 2007; Goldman et al. 2010; Wahhaj et al. 2011; Bowler et al. 2013; Naud et al. 2014; Gauza et al. 2015; Smith et al. 2015; Bowler et al. 2017) as stellar age is known to be a relevant parameter for the spectral and photometric characterization of substellar objects. HR 2562 B sits at the L-T transition, very near the location of (although slightly redder than) HN Peg B, which has comparable age (300-400 Myr) and mass $\left(\sim 20 M_{\text {Jup }}\right)$, according to Luhman et al. (2007).

\subsubsection{Companion parameters through evolutionary models}

We estimated the main parameters of the companion using the AMES-COND models (Allard et al. 2003) and the AMESDUSTY models (Allard et al. 2001), starting from photometry reported in Table 3 and assuming the age discussed in Sect. 2. The results for the companion mass and its surface gravity are listed in Tables 4 and 5. For these calculations, we adopted three different ages that cover the whole age range proposed in Sect. 2 for HR 2562. The $Y$ and $J$ band results are in good agreement with each other, while the $H$ band determination tends to provide different masses, especially in the case of the AMES-COND models. For the intermediate age of $450 \mathrm{Myr}$ the AMES-COND model provides a value of the mass between 20 and $30 M_{\text {Jup }}(\log g \sim 4.7$ dex, with $g$ in $\operatorname{cgs})$, which is slightly lower than that obtained by Konopacky et al. (2016) because of the younger age adopted in this paper. On the other

\footnotetext{
4 http://www.eso.org/observing/etc/bin/gen/ form?INS . MODE=swspectr+INS . NAME=SKYCALC
}

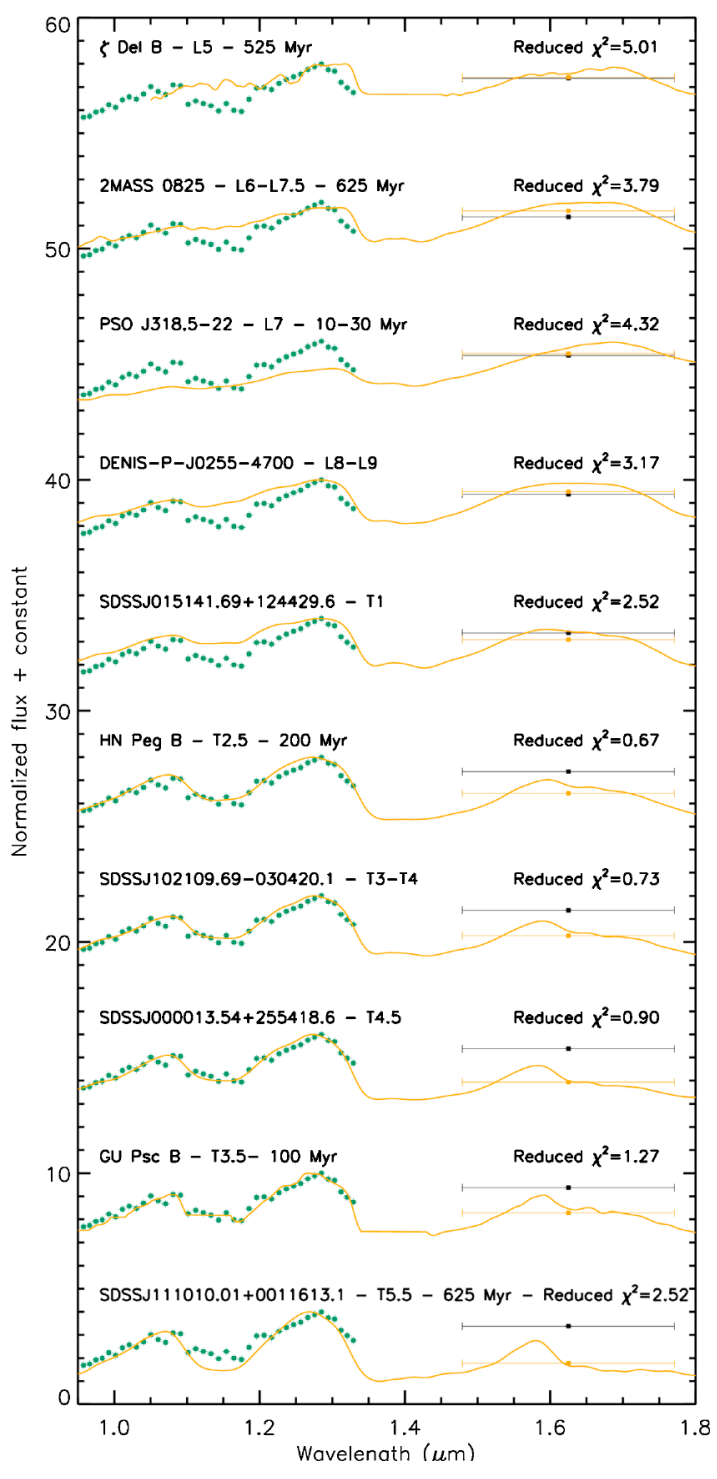

Fig. 6. Comparison of the extracted spectrum of HR 2562 B (green squares) to those of ten template spectra (orange lines) with spectral types ranging between L5 and T5.5. The dark square represents the value of the IRDIS $\mathrm{H}$ broadband photometry, while the orange square is the $\mathrm{H}$ broadband photometry for the compared objects calculated starting from the original spectrum.

Table 4. Estimation of the companion mass (in $M_{\mathrm{Jup}}$ ) and $\log (g)$ using $Y, J$, and $H$ band photometry and the AMES-COND models.

\begin{tabular}{ccccccc}
\hline \hline Age & \multicolumn{3}{c}{ Mass $\left(M_{\text {Jup }}\right)$} & \multicolumn{3}{c}{$\log (g)$} \\
(Myr) & $Y$ & $J$ & $H$ & $Y$ & $J$ & $H$ \\
\hline 200 & 11.46 & 11.80 & 18.55 & 4.34 & 4.35 & 4.58 \\
450 & 20.85 & 22.12 & 29.16 & 4.69 & 4.71 & 4.85 \\
750 & 27.40 & 28.67 & 37.58 & 4.85 & 4.87 & 5.01 \\
\hline
\end{tabular}

hand, the AMES-DUSTY model provides a larger value of 40$46 M_{\text {Jup }}(\log g \sim 5.0 \mathrm{dex})$. These values for the mass are in good agreement with those recently found by Dupuy \& Liu (2017), who evaluated the dynamical masses of ultracool dwarfs and found a mass range of around 30-40 $M_{\text {Jup }}$ for BDs with spectral type around T2-T3 (see Sect. 5.1.3). 
D. Mesa et al.: New spectro-photometric characterization of the substellar object HR 2562 B using SPHERE
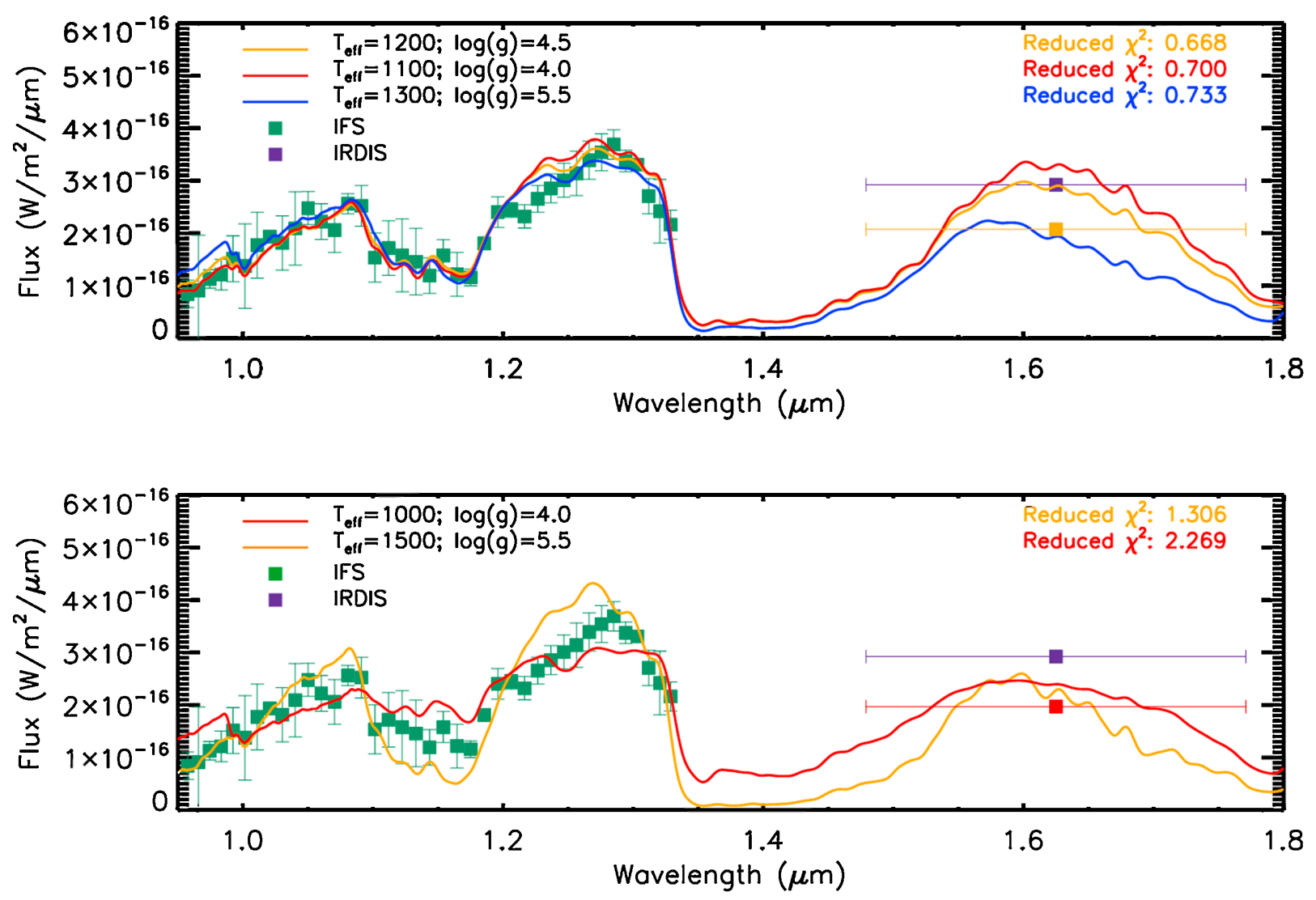

Fig. 7. Upper panel: Spectrum extracted for HR 2562 B (green squares) with its error bars. The colored solid lines represents the three best fitting spectra from the BT-Settl synthetic spectra. The orange square is obtained from the corresponding spectrum calculating the flux on the IRDIS H broadband filter in order to better compare it with the IRDIS data. The same operation was not done for the other spectra to avoid confusion in the final plot. Lower panel: Same as upper panel, but using synthetic spectra with poorer fits.

Table 5. Estimation of the companion mass (in $M_{\text {Jup }}$ ) and $\log (g)$ using $Y, J$, and $H$ band photometry and the AMES-DUSTY models.

\begin{tabular}{ccccccc}
\hline \hline \multirow{2}{*}{ Age } & \multicolumn{3}{c}{ Mass $\left(M_{\text {Jup }}\right)$} & \multicolumn{3}{c}{$\log (g)$} \\
(Myr) & $Y$ & $J$ & $H$ & $Y$ & $J$ & $H$ \\
\hline 200 & 34.04 & 32.14 & 28.41 & 4.82 & 4.80 & 4.74 \\
450 & 46.59 & 44.84 & 40.21 & 5.03 & 5.02 & 4.97 \\
750 & 56.08 & 54.34 & 49.51 & 5.18 & 5.16 & 5.12 \\
\hline
\end{tabular}

\subsubsection{Fitting with spectral template}

In order to better ascertain the spectral type of HR $2562 \mathrm{~B}$, we performed fit procedures of its spectrum with sample spectra of field BDs taken from the Spex Prism spectral Libraries ${ }^{5}$ (Burgasser 2014). The final result is displayed in Fig. 6, where we show the comparison of the extracted spectrum for HR $2562 \mathrm{~B}$ with those of ten objects having spectral types between L5 and T5 (Burgasser et al. 2004, 2006; Luhman et al. 2007; Cushing et al. 2008; Stephens et al. 2009; Liu et al. 2013; De Rosa et al. 2014; Naud et al. 2014; Gagné et al. 2015). The best fit was obtained with the spectrum of HN Peg B (Luhman et al. 2007), which is classified as spectral type T2.5. This result confirms what we obtained in Sect. 5.1.1 where we showed that the positions of these two objects are very similar in the colormagnitude diagram (see Fig. 5). Thus, we found compelling

\footnotetext{
5 http://pono.ucsd.edu/ adam/browndwarfs/spexprism/
}

evidence that they could be very similar objects. However, a comparably good fit was also obtained with the spectrum of SDSS J143553.25+112948.6 (Chiu et al. 2006), which was classified as spectral type T2 \pm 1 , and SDSS J102109.69030420.1AB (Burgasser et al. 2006), which was classified as spectral type T3. From these results we can define this object as a T2-T3 spectral type. It is noteworthy, however, that the value derived from the IRDIS $\mathrm{H}$ broadband data does not agree well with this result. Indeed, looking at the comparison of the flux in $\mathrm{H}$ broadband between HR $2562 \mathrm{~B}$ and the template spectra represented by the dark and the orange squares in Fig. 6, respectively, our finding seems in better agreement with a late-L spectral type.

\subsubsection{Fitting with atmospheric models}

To further constrain the physical parameters of HR 2562 B we compared its extracted spectrum to synthetic spectra from different atmospheric models.

We first used the BT-Settl models (Allard 2014) with a grid covering $T_{\text {eff }}$ between 900 and $2500 \mathrm{~K}$ with a step of $100 \mathrm{~K}$, and a $\log g$ ranging between 2.5 and 5.5 dex with a step of 0.5. All the models were of solar metallicity. The results of this procedure are displayed in Fig. 7. The best fit is for the spectrum with $T_{\text {eff }}=1200 \mathrm{~K}$ and $\log g=4.5$; however, a good fit is obtained even for a spectrum with $T_{\text {eff }}=1100 \mathrm{~K}$ and $\log g=4.0$, and for a spectrum with $T_{\text {eff }}=1300 \mathrm{~K}$ and $\log g=5.5$. We can then conclude that the fit gives $T_{\text {eff }}=1200 \pm 100 \mathrm{~K}$ and $\log g=4.5_{-0.5}^{1.0}$. The $T_{\text {eff }}$ and $\log (g)$ values found through 

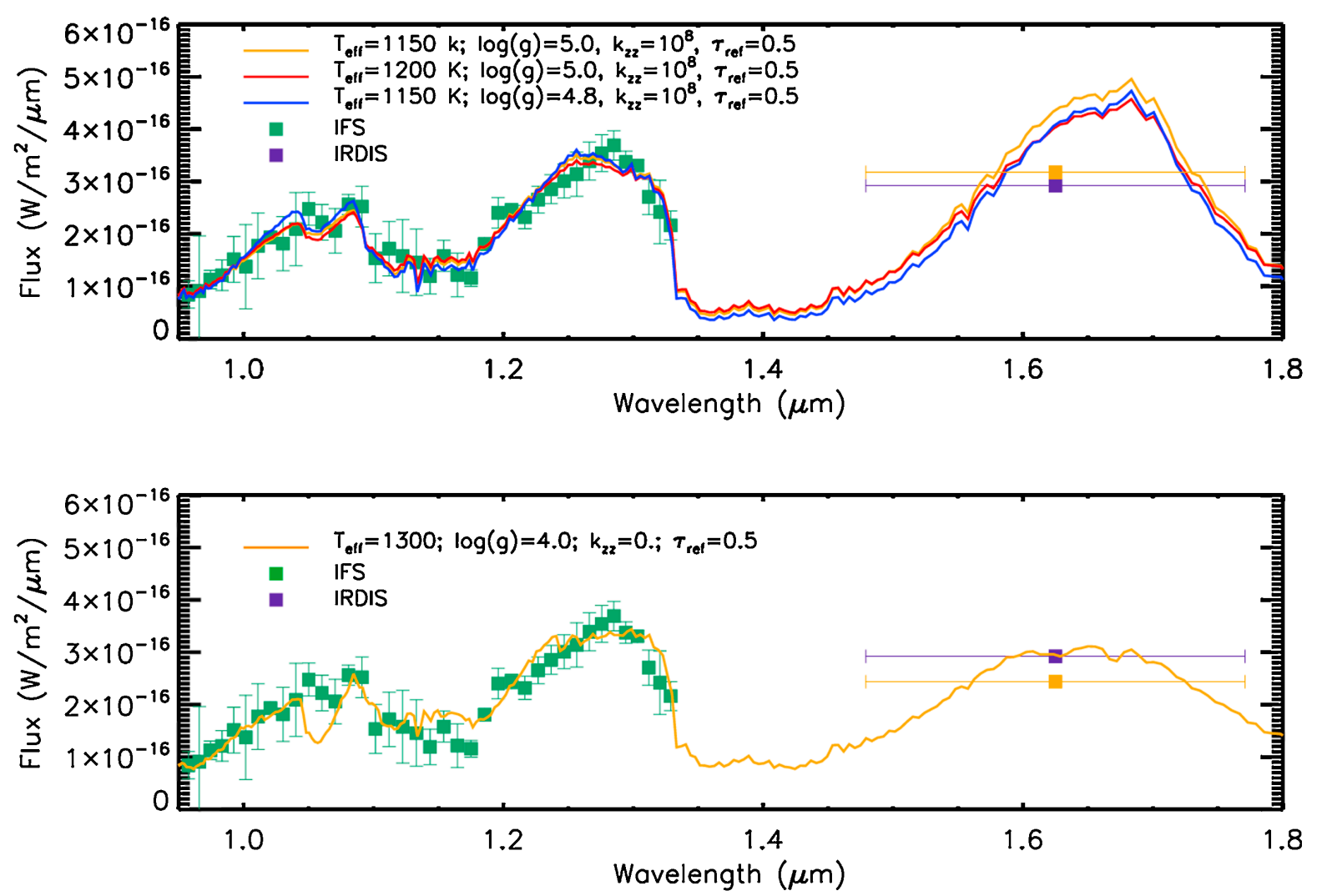

Fig. 8. Same as Fig. 7, but for the case of the Exo-REM models. The poorer fit in the lower panel is the best case taking into account equilibrium chemistry.
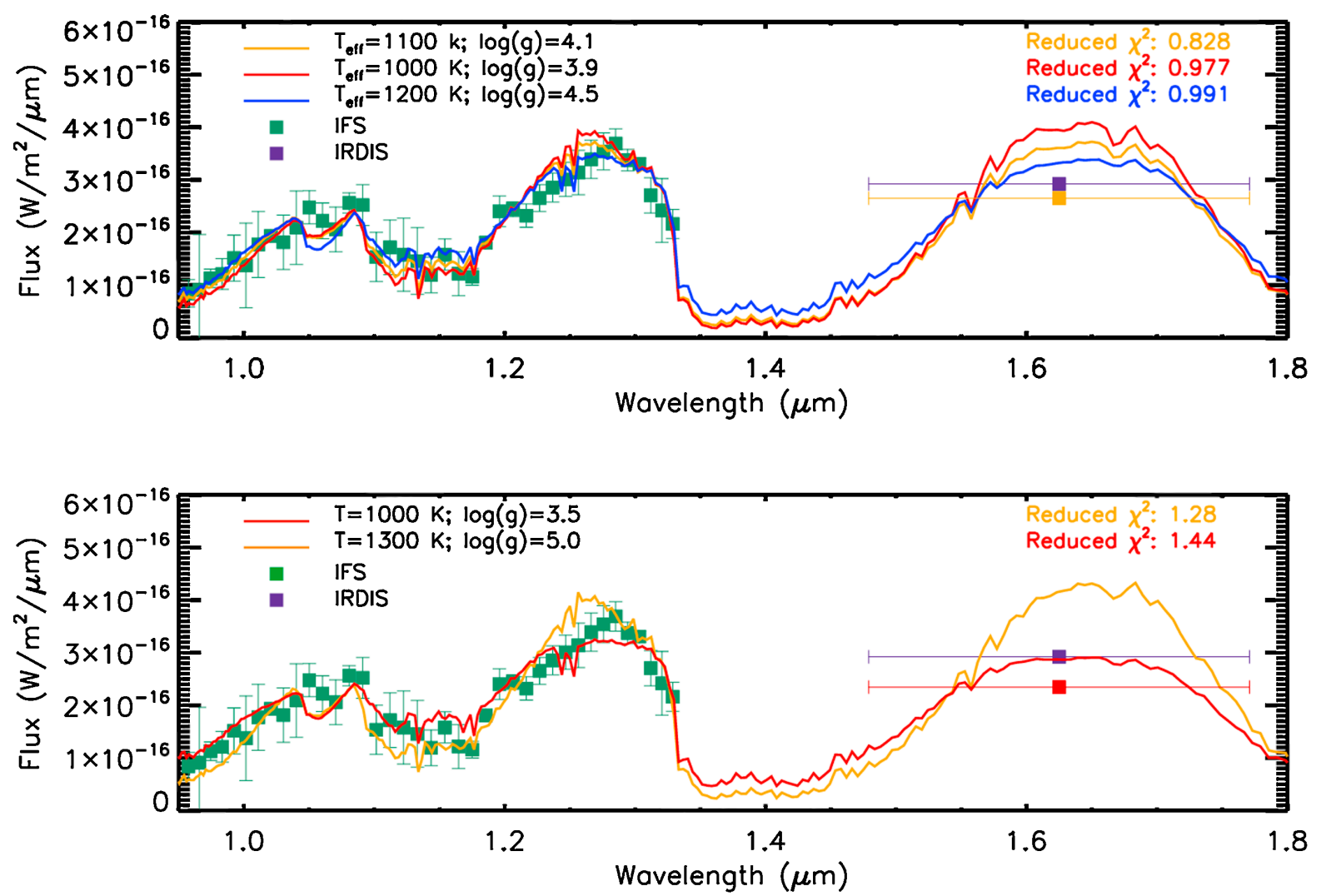

Fig. 9. Same as Fig. 7, but for the case of the Exo-REM models with modified cloud treatment. 

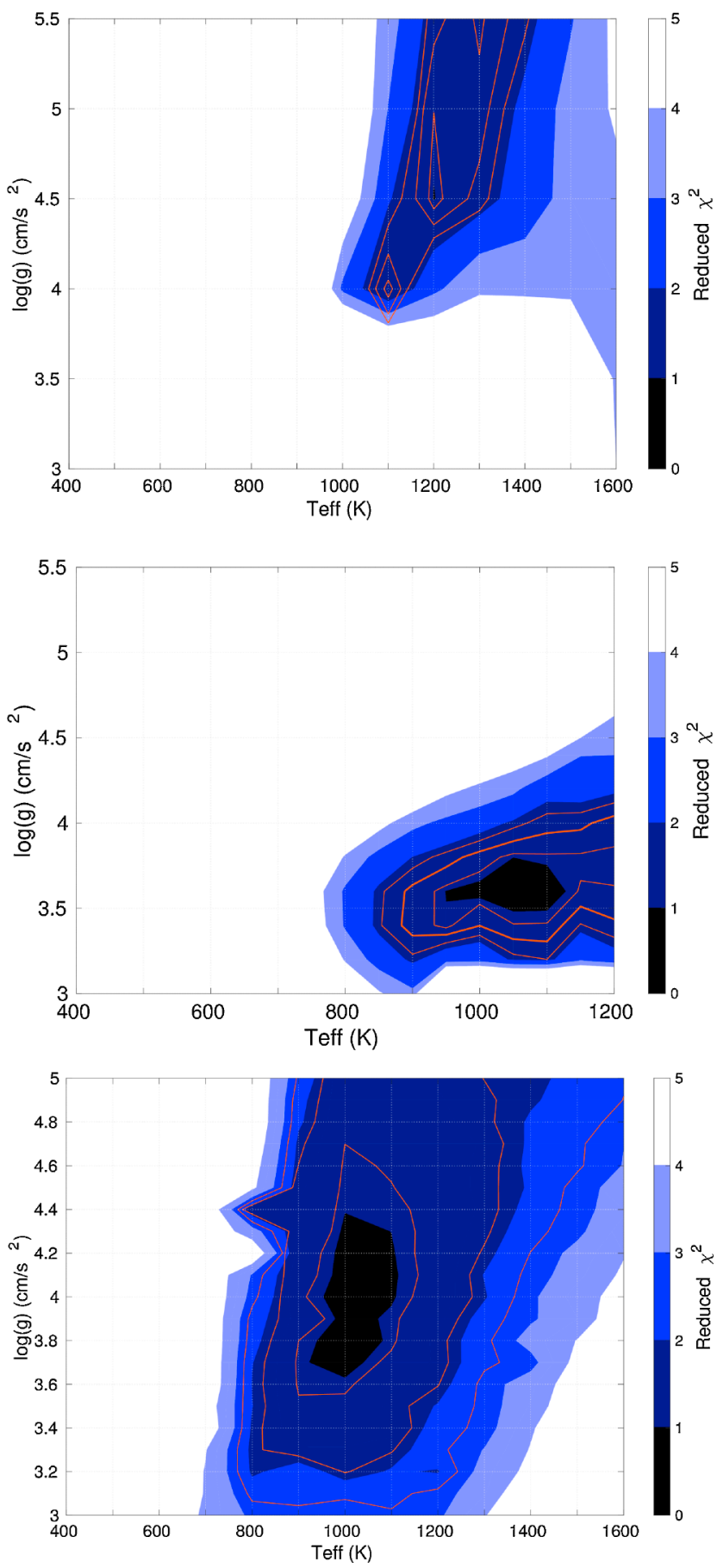

Fig. 10. Reduced $\chi^{2}$ contour map of the BT-Settl (top), Exo-REM (middle), and Exo-REM modified cloud (bottom) models. All the plots shown here are for solar metallicities. The red contours are for 1,3 , and $5 \sigma$.

this procedure are in good agreement with those found by Konopacky et al. (2016).

\subsubsection{Comparison of results from evolutionary and atmospheric models}

As a second step, we compared the extracted spectrum for HR 2562 B with the Exo-REM model (Baudino et al. 2015), which is specifically developed for young giant exoplanets. We first generated a grid of models with $T_{\text {eff }}$ between 400 and $1800 \mathrm{~K}$ with a step of $100 \mathrm{~K}, \log g$ in the range 2.5-5.5 dex with a step

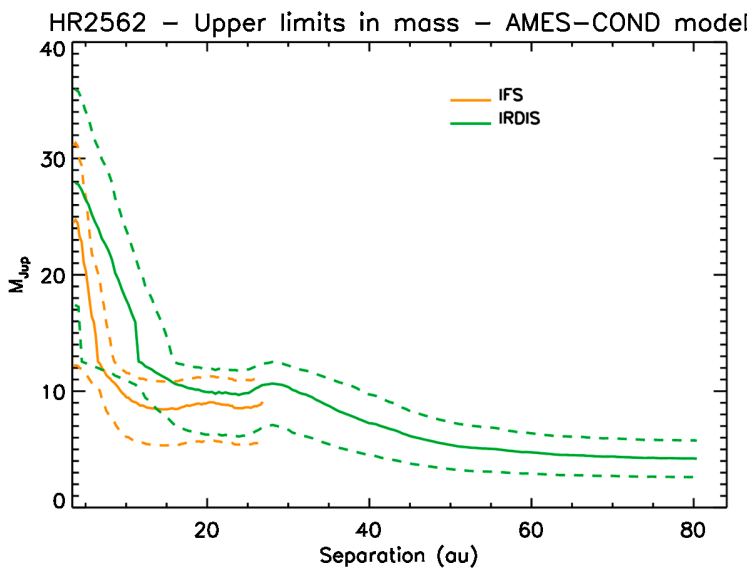

Fig. 11. Mass limits for possible companions around HR 2562. The green lines are from the IRDIS data, while the orange lines are from the IFS data. The solid lines were obtained assuming an age of $450 \mathrm{Myr}$, while the dashed lines were obtained assuming the lower and upper limits of the age range for this star: 200 and $750 \mathrm{Myr}$.

of 0.1 , with and without $\mathrm{Fe}$ and $\mathrm{Mg}_{2} \mathrm{SiO}_{4}$ considering a particle radius of $30 \mu \mathrm{m}$, and assuming equilibrium and nonequilibrium chemistry $\left(k_{z z}=10^{8} \mathrm{~cm}^{2} \mathrm{~s}^{-1}\right)$. All these models were then compared to the spectrum of HR $2562 \mathrm{~B}$ to find the one that minimized the $\chi^{2}$. All the values with $\chi^{2}$ less than 1 were then considered as acceptable. The best fits obtained from this procedure are shown in the upper panel of Fig. 8; as a comparison we show an example of a poor quality fit in the lower panel. The best results are for models with $T_{\text {eff }}$ in the range 1100-1200 K, $\log g \sim 5.0$ dex, cloud with an optical depth of $\tau_{r e f}=0.5$, and nonequilibrium chemistry. However, considering all the models with a value of $\chi^{2}$ less than 1 we can only constrain a $T_{\text {eff }}$ in the range $950-1200 \mathrm{~K}$ and $\log g$ in the range 3.4-5.2 dex.

In order to carefully evaluate the contribution of the cloud coverage to the definition of the main parameters for the companion, we also used a new version of the Exo-REM models. This includes a self-consistent modeling of the clouds (both iron and silicate) that is based on the parameterization of the $k_{z z}$ profile, and considers cloud condensation, coalescence, sedimentation, and vertical mixing (Charnay et al., in prep.). The size of the cloud particles is calculated by comparing typical timescales for condensation, coalescence, sedimentation, and vertical mixing, following the method described in Rossow (1978). Moreover, these models take into account cloud scattering and can also simulate a cloud coverage fraction, as done by Marley et al. (2010). The model $T_{\text {eff }}$ ranges from 400 to $2000 \mathrm{~K}$ with a step of $100 \mathrm{~K}$, while $\log g$ varies from 3 to 5 dex with a step of 0.1 dex. We considered models with $0.3,1$, and 3 times the solar metallicity. We decided to explore different metallicities because we found that the star is slightly metal rich with respect to the Sun (see Sect. 2.1) and because there are claims in the literature about metallicity enhancement of substellar companions with respect to their parent stars (Skemer et al. 2016; Samland et al. 2017). Equilibrium and nonequilibrium chemistry were adopted along with different cloud coverage: the best fitting results in models with nonequilibrium chemistry and a cloud coverage of $95 \%$. The best fit spectra from this procedure are shown in Fig. 9, while the $\chi^{2}$ map is displayed in Fig. 10 together with those from BT-Settl and not modified Exo-REM models. The result of this second procedure points toward an object at $T_{\text {eff }}$ between 1000 and $1200 \mathrm{~K}$ and $\log g$ just above 4.0 dex. Indeed, considering a metallicity three times the solar value, we obtain 
$T_{\text {eff }}=1100 \pm 100 \mathrm{~K}$ and $\log g=4.3 \pm 0.6 \mathrm{dex}$, while for a solar metallicity we estimate from the model $T_{\text {eff }}=900-1150 \mathrm{~K}$ and $\log g=4.1 \pm 0.6 \mathrm{dex}$. Instead, for the exploration of the metallicity, no definitive results can be deduced from these models even if models with metallicity lower than solar tend to have values of $\log g$ less than 4, while models with metallicity higher than solar tend to have values of $\log g$ nearer to the values deduced through evolutionary models.

As for $\log g$, by comparing our data with atmospheric models we have inferred values lower than those obtained from the evolutionary models, in particular when DUSTY models are employed (see Sect. 5.1.2). The explanation is that the cloud coverage of the atmosphere of HR 2562 B is likely only partial, as confirmed by the quite compelling evidence provided by the Exo-REM models. However, when we consider all of the atmospheric model datasets we obtain a wide range of $\log g$ ranging from 3.4 to 5.2. We conclude that it is difficult to firmly constrain the value of $\log g$ by exploiting atmospheric models because their dependence on gravity is relatively small. From this point of view, the estimates that we gathered using the evolutionary models are much more useful, and show significantly smaller dispersion. In fact, they represents two extreme conditions (absence of clouds in the COND models vs complete coverage of clouds for the DUSTY case) that can be used to constrain the correct value of $\log g$. We can then consider the lower and the upper values obtained from these models and listed in Tables 4 and 5 and use them to estimate the value of $\log g$ as $4.76 \pm 0.42$. The main contribution to the error bar is clearly given by the uncertainties on the age of the system. On the other hand, $T_{\text {eff }}$ is much better constrained with values in the range $900-1300 \mathrm{~K}$ so that we can then assume for $T_{\text {eff }}$ a value of $1100 \pm 200 \mathrm{~K}$. This is in good agreement with the spectral classification of T2-T3 that we obtained from the procedure described in Sect. 5.1.3. Indeed, Pecaut \& Mamajek (2013) foresee a value of $T_{\text {eff }}$ of $1200 \mathrm{~K}$ for a T2 spectral type and of $1160 \mathrm{~K}$ for a T3 spectral type.

\subsection{Mass limits for other objects in the system}

Starting from the contrast limit displayed in Fig. 3 and using the AMES-COND models (Allard et al. 2003), we obtained the limit in mass for other possible companions around HR 2562. This is shown in Fig. 11, where the solid lines are obtained assuming an age of 450 Myr. Moreover, with the aim of showing the dependency of the limits on the age, we display in dashed lines the mass limits that are obtained assuming 200 and $750 \mathrm{Myr}$ (these are the lower and upper limits proposed for the age). From our findings, we should be able to see objects with mass on the order of $10 M_{\text {Jup }}$ at separations larger than $10 \mathrm{au}$, while at separations larger than 40 au IRDIS should allow us to detect objects with masses of a few $M_{\text {Jup }}$.

\section{Conclusions}

In this paper we have presented the high-contrast imaging observations of the star HR 2562 obtained with SPHERE. We were able to recover the low-mass companion previously discovered by Konopacky et al. (2016) both with IRDIS and IFS.

Using the companion photometry extracted in the $Y, J$, and $H$ band and the AMES-COND evolutionary models, we derived a mass in the 20-30 $M_{\text {Jup }}$ range and a $\log g \sim 4.7$ dex. Conversely, by adopting the AMES-DUSTY models we obtained larger masses $\left(\gtrsim 40 M_{\text {Jup }}\right)$ and $\log g$ of $\approx 5.0$ dex.
The spectro-photometric measurements performed with IFS allowed us to extract a low-resolution spectrum of the companion in $Y$ and $J$ band, while IRDIS provided us $\mathrm{H}$ broadband photometry. Fitting the extracted spectrum with a sample of spectra of low-mass objects, we were able to classify HR 2562 B as an early $\mathrm{T}$ (T2-T3) spectral type, although the $\mathrm{H}$ broadband photometry seems more in accordance with late-L spectra. Our spectral classification is then not in complete agreement with that of Konopacky et al. (2016). The IFS spectrum is very similar to that obtained by GPI in the $J$ band so that the different spectral classification is probably due to the different spectral coverage of the two instruments outside the $J$ band. In fact, the extension to the $Y$ band allows SPHERE to see the peak at $1.08 \mu \mathrm{m}$ (not visible in GPI), making it possible to identify the early-T nature of the object. On the other hand, the GPI spectrum of Konopacky et al. (2016) extends to the $H$ and $K$ band; their classification was mainly based on the $H$ and $K$ band observations. Thus, while the presence of the peak at $1.08 \mu \mathrm{m}$ in the IFS spectrum strongly points towards an early-T type spectrum, several uncertainties still remain and observations on a wider wavelength range are badly needed to completely disentangle the companion spectral classification.

It is also possible that some variability affects the spectral appearance of HR $2562 \mathrm{~B}$. Indeed, photometric variability due to nonuniform cloud coverage has been shown to be more prominent for L/T transition objects (see, e.g., Buenzli et al. 2014; Metchev et al. 2015).

The use of synthetic spectra from atmospheric models allowed us to put some constraints on the physical parameters of the companion. Using the BT-Settl models, the best solution was found for $T_{\text {eff }}=1200 \pm 100 \mathrm{~K}$ and $\log g=4.5 \pm 0.5 \mathrm{dex}$. The Exo-REM models allowed us to define a range of 950-1200 K for $T_{\text {eff }}$ and of 3.4-5.2 for $\log g$, with strong indications for the upper limit of these intervals. Finally, for our analysis we used a new version of the Exo-REM models that were modified to have a self-consistent treatment of the clouds. In this case our findings suggest a $T_{\text {eff }}$ in the range $1000-1200 \mathrm{~K}$ and a $\log g$ lower than that found by the other models (on the order of $\approx 4.0 \mathrm{dex}$ ). However, the two Exo-REM models both give strong hints of the presence of clouds with a high cloud coverage (best fit of 95\%) and nonequilibrium chemistry.

Summarizing all the results described in the paper, HR 2562 B should have a mass of $32 \pm 14 M_{\text {Jup }}$ with $T_{\text {eff }}=1100 \pm 200 \mathrm{~K}$ and $\log g=4.75 \pm 0.41$.

Acknowledgements. The authors thanks Quinn Konopacky for sharing the GPI spectra of HR 2562 B. We are grateful to the SPHERE team and all the people at Paranal for the great effort during the SPHERE GTO run. This work has made use of the SPHERE Data Center, jointly operated by OSUG/IPAG (Grenoble), PYTHEAS/LAM/CeSAM (Marseille), OCA/Lagrange (Nice), and Observatoire de Paris/LESIA (Paris). D.M. acknowledges support from the ESO-Government of Chile Joint Comittee program "Direct imaging and characterization of exoplanets". D.M., A.Z., V.D.O., R.G., R.U.C., S.D., and C.L. acknowledge support from the "Progetti Premiali" funding scheme of the Italian Ministry of Education, University, and Research. J.H. is supported by the French ANR through the GIPSE grant ANR-14-CE33-0018. This work has been supported by the project PRIN-INAF 2016 The Cradle of Life - GENESIS-SKA (General Conditions in Early Planetary Systems for the rise of life with SKA). We acknowledge support from the French National Research Agency (ANR) through the GUEPARD project grant ANR10-BLANC0504-01. SPHERE is an instrument designed and built by a consortium consisting of IPAG (Grenoble, France), MPIA (Heidelberg, Germany), LAM (Marseille, France), LESIA (Paris, France), Laboratoire Lagrange (Nice, France), INAF- Osservatorio di Padova (Italy), Observatoire de Genève (Switzerland), ETH Zurich (Switzerland), NOVA (Netherlands), ONERA (France), and ASTRON (Netherlands) in collaboration with ESO. SPHERE was funded by ESO, with additional contributions from CNRS (France), MPIA (Germany), INAF (Italy), FINES (Switzerland), and NOVA (Netherlands). SPHERE also received funding from the European 
Commission Sixth and Seventh Framework Programmes as part of the Optical Infrared Coordination Network for Astronomy (OPTICON) under grant number RII3-Ct-2004-001566 for FP6 (2004-2008), grant number 226604 for FP7 (2009-2012), and grant number 312430 for FP7 (2013-2016). This research has benefited from the SpeX Prism Spectral Libraries, maintained by Adam Burgasser at http://pono.ucsd.edu/ adam/browndwarfs/spexprism

\section{References}

Allard, F. 2014, in Exploring the Formation and Evolution of Planetary Systems, eds. M. Booth, B. C. Matthews, \& J. R. Graham, IAU Symp., 299, 271

Allard, F., Hauschildt, P. H., Alexander, D. R., Tamanai, A., \& Schweitzer, A. 2001, ApJ, 556, 357

Allard, F., Guillot, T., Ludwig, H.-G., et al. 2003, in Brown Dwarfs, ed. E. Martín, IAU Symp., 211, 325

Allard, F., Homeier, D., \& Freytag, B. 2012, Phil. Trans. Roy. Soc. Lond. Ser. A, 370,2765

Andersen, J., Nordstrom, B., Ardeberg, A., et al. 1985, A\&AS, 59, 15

Asiain, R., Figueras, F., Torra, J., \& Chen, B. 1999, A\&A, 341, 427

Bailey, V., Meshkat, T., Reiter, M., et al. 2014, ApJ, 780, L4

Balachandran, S. 1995, ApJ, 446, 203

Baudino, J.-L., Bézard, B., Boccaletti, A., et al. 2015, A\&A, 582, A83

Beuzit, J.-L., Feldt, M., Dohlen, K., et al. 2008, in Ground-based and Airborne Instrumentation for Astronomy II, Proc. SPIE, 7014, 701418

Biller, B. A., Liu, M. C., Wahhaj, Z., et al. 2010, ApJ, 720, L82

Boesgaard, A. M., \& Tripicco, M. J. 1986, ApJ, 302, L49

Boesgaard, A. M., Budge, K. G., \& Ramsay, M. E. 1988, ApJ, 327, 389

Bohlin, R. C. 2007, in The Future of Photometric, Spectrophotometric and Polarimetric Standardization, eds. C. Sterken, ASP Conf. Ser., 364, 315

Bonnefoy, M. 2015, in AAS/Division for Extreme Solar Systems Abstracts, 3, 203.05

Bonnefoy, M., Zurlo, A., Baudino, J. L., et al. 2016, A\&A, 587, A58

Bowler, B. P., Liu, M. C., Shkolnik, E. L., \& Dupuy, T. J. 2013, ApJ, 774, 55

Bowler, B. P., Liu, M. C., Mawet, D., et al. 2017, AJ, 153, 18

Bressan, A., Marigo, P., Girardi, L., et al. 2012, MNRAS, 427, 127

Buenzli, E., Apai, D., Radigan, J., Reid, I. N., \& Flateau, D. 2014, ApJ, 782, 77

Burgasser, A. J. 2014, in Astron. Soc. India Conf. Ser., 11, 7

Burgasser, A. J., McElwain, M. W., Kirkpatrick, J. D., et al. 2004, AJ, 127, 2856

Burgasser, A. J., Geballe, T. R., Leggett, S. K., Kirkpatrick, J. D., \& Golimowski, D. A. 2006, ApJ, 637, 1067

Casagrande, L., Schönrich, R., Asplund, M., et al. 2011, A\&A, 530, A138

Chauvin, G., Lagrange, A.-M., Dumas, C., et al. 2004, A\&A, 425, L29

Chauvin, G., Lagrange, A.-M., Zuckerman, B., et al. 2005, A\&A, 438, L29

Chen, C. H., Mittal, T., Kuchner, M., et al. 2014, ApJS, 211, 25

Chilcote, J., Pueyo, L., De Rosa, R. J., et al. 2017, AJ, 153, 182

Chiu, K., Fan, X., Leggett, S. K., et al. 2006, AJ, 131, 2722

Claudi, R. U., Turatto, M., Gratton, R. G., et al. 2008, in SPIE Conf. Ser., 7014

Cushing, M. C., Marley, M. S., Saumon, D., et al. 2008, ApJ, 678, 1372

da Silva, L., Girardi, L., Pasquini, L., et al. 2006, A\&A, 458, 609

de Medeiros, J. R., \& Mayor, M. 1999, A\&AS, 139, 433

De Rosa, R. J., Patience, J., Ward-Duong, K., et al. 2014, MNRAS, 445, 3694

De Rosa, R. J., Rameau, J., Patience, J., et al. 2016, ApJ, 824, 121

Delorme, P., Schmidt, T., Bonnefoy, M., et al. 2017 A\&A, 608, A79

Desidera, S., Covino, E., Messina, S., et al. 2011, A\&A, 529, A54

Desidera, S., Covino, E., Messina, S., et al. 2015, A\&A, 573, A126

Dohlen, K., Langlois, M., Saisse, M., et al. 2008, in SPIE Conf. Ser., 7014

D’Orazi, V., Desidera, S., Gratton, R. G., et al. 2017, A\&A, 598, A19

Dupuy, T. J., \& Liu, M. C. 2017, ApJS, 231, 15

Gagné, J., Lafrenière, D., Doyon, R., Malo, L., \& Artigau, É. 2014, ApJ, 783, 121

Gagné, J., Burgasser, A. J., Faherty, J. K., et al. 2015, ApJ, 808, L20
Garcia, E. V., Currie, T., Guyon, O., et al. 2017, ApJ, 834, 162

Gauza, B., Béjar, V. J. S., Pérez-Garrido, A., et al. 2015, ApJ, 804, 96

Goldman, B., Marsat, S., Henning, T., Clemens, C., \& Greiner, J. 2010, MNRAS, 405,1140

Gray, R. O., Corbally, C. J., Garrison, R. F., et al. 2006, AJ, 132, 161

Johnson-Groh, M., Marois, C., De Rosa, R. J., et al. 2017, AJ, 153, 190

Jones, A., Noll, S., Kausch, W., Szyszka, C., \& Kimeswenger, S. 2013, A\&A, 560, A91

Konopacky, Q. M., Rameau, J., Duchêne, G., et al. 2016, ApJ, 829, L4

Lagrange, A.-M., Bonnefoy, M., Chauvin, G., et al. 2010, Science, 329, 57

Langlois, M., Vigan, A., Moutou, C., et al. 2013, in Proc. Third AO4ELT Conf., eds. S. Esposito \& L. Fini, 63

Liu, M. C., Magnier, E. A., Deacon, N. R., et al. 2013, ApJ, 777, L20

Luhman, K. L., Patten, B. M., Marengo, M., et al. 2007, ApJ, 654, 570

Macintosh, B., Graham, J. R., Ingraham, P., et al. 2014, Proc. Natl. Acad. Sci., 111,12661

Macintosh, B., Graham, J. R., Barman, T., et al. 2015, Science, 350, 64

Maire, A.-L., Bonnefoy, M., Ginski, C., et al. 2016, A\&A, 587, A56

Marley, M. S., Saumon, D., \& Goldblatt, C. 2010, ApJ, 723, L117

Marois, C., Lafrenière, D., Doyon, R., Macintosh, B., \& Nadeau, D. 2006a, ApJ, 641,556

Marois, C., Lafrenière, D., Macintosh, B., \& Doyon, R. 2006b, ApJ, 647, 612

Marois, C., Macintosh, B., Barman, T., et al. 2008, Science, 322, 1348

Marois, C., Correia, C., Galicher, R., et al. 2014, in Adaptive Optics Systems IV, Proc. SPIE, 9148, 91480U

Mesa, D., Gratton, R., Zurlo, A., et al. 2015, A\&A, 576, A121

Mesa, D., Vigan, A., D’Orazi, V., et al. 2016, A\&A, 593, A119

Metchev, S. A., Heinze, A., Apai, D., et al. 2015, ApJ, 799, 154

Milli, J., Hibon, P., Christiaens, V., et al. 2017, A\&A, 597, L2

Montes, D., López-Santiago, J., Gálvez, M. C., et al. 2001, MNRAS, 328, 45

Moór, A., Ábrahám, P., Derekas, A., et al. 2006, ApJ, 644, 525

Moór, A., Pascucci, I., Kóspál, Á., et al. 2011, ApJS, 193, 4

Moór, A., Kóspál, Á., Ábrahám, P., et al. 2015, MNRAS, 447, 577

Naud, M.-E., Artigau, É., Malo, L., et al. 2014, ApJ, 787, 5

Noll, S., Kausch, W., Barden, M., et al. 2012, A\&A, 543, A92

Nordström, B., Mayor, M., Andersen, J., et al. 2004, A\&A, 418, 989

Pace, G. 2013, A\&A, 551, L8

Pavlov, A., Möller-Nilsson, O., Feldt, M., et al. 2008, in SPIE Conf. Ser., 7019, 39

Pecaut, M. J., \& Mamajek, E. E. 2013, ApJS, 208, 9

Rajan, A., Rameau, J., De Rosa, R. J., et al. 2017, AJ, 154, 10

Rameau, J., Chauvin, G., Lagrange, A.-M., et al. 2013, ApJ, 779, L26

Rhee, J. H., Song, I., Zuckerman, B., \& McElwain, M. 2007, ApJ, 660, 1556

Rossow, W. B. 1978, Icarus, 36, 1

Samland, M., Mollière, P., Bonnefoy, M., et al. 2017, A\&A, 603, A57

Sivaramakrishnan, A., \& Oppenheimer, B. R. 2006, ApJ, 647, 620

Skemer, A. J., Morley, C. V., Zimmerman, N. T., et al. 2016, ApJ, 817, 166

Smith, L. C., Lucas, P. W., ContrerasPeña, C., et al. 2015, MNRAS, 454, 4476

Soummer, R., Pueyo, L., \& Larkin, J. 2012, ApJ, 755, L28

Stauffer, J., Rebull, L., Bouvier, J., et al. 2016, AJ, 152, 115

Steinhauer, A., \& Deliyannis, C. P. 2004, ApJ, 614, L65

Stephens, D. C., Leggett, S. K., Cushing, M. C., et al. 2009, ApJ, 702, 154

Valenti, J. A., \& Piskunov, N. 1996, A\&AS, 118, 595

Vigan, A., Moutou, C., Langlois, M., et al. 2010, MNRAS, 407, 71

Vigan, A., Bonnefoy, M., Ginski, C., et al. 2016, A\&A, 587, A55

Wahhaj, Z., Liu, M. C., Biller, B. A., et al. 2011, ApJ, 729, 139

Zuckerman, B., \& Song, I. 2004, ARA\&A, 42, 685

Zurlo, A., Vigan, A., Mesa, D., et al. 2014, A\&A, 572, A85

Zurlo, A., Vigan, A., Galicher, R., et al. 2016, A\&A, 587, A57 